\title{
Quantitative MRI data in Multiple Sclerosis patients: a pattern recognition study
}

\author{
Rodrigo Antonio Pessini ${ }^{1 *}$, Antonio Carlos dos Santos ${ }^{1}$, Carlos Ernesto Garrido Salmon ${ }^{2}$ \\ ${ }^{1}$ Center of Imaging Sciences and Medical Physics, Clinics Hospital, Ribeirão Preto Medical School, University of São Paulo, \\ Ribeirão Preto, SP, Brazil. \\ ${ }^{2}$ Inbrain Lab, Department of Physics, Faculty of Philosophy, Sciences and Letters of Ribeirão Preto, University of São Paulo, \\ Ribeirão Preto, SP, Brazil.
}

\begin{abstract}
Introduction: Multiple Sclerosis (MS) is a neurodegenerative disease characterized by inflammatory demyelination in the central nervous system. Quantitative Magnetic Resonance Imaging (qMRI) enables a detailed characterization of brain tissue, but generates a large number of numerical results. In this study, we elucidated the main qMRI techniques and the brain regions that allow the identification of MS patients from neuroimaging data and pattern recognition techniques. Methods: The data came from the combination of computational tools of image processing and neuroimaging acquired in a 3 Tesla scanner using different techniques: Diffusion, T2 Relaxometry, Magnetization Transfer Ratio (MTR) and Structural Morphometry. Data from 126 brain regions of 203 healthy individuals and $124 \mathrm{MS}$ patients were separated into two groups and processed in a data-mining program using the k-nearest-neighbor $(\mathrm{KNN})$ algorithm. Results: The most relevant anatomical structures in the classification procedure were: corpus callosum, precuneus, left cerebellum and fusiform. Among the quantitative techniques the most relevant was the MTR, being indicated for longitudinal studies of this disease. KNN with 5 neighbors and pre-selected attributes had a better performance with an area under the ROC curve $(97.3 \%)$ and accuracy $(95.7 \%)$. A restricted classification considering only brain regions previously reported in the literature as affected by MS brought slightly lower scores, area: $97.1 \%$ and accuracy: $93.2 \%$. Conclusion: The use of standard recognition techniques from quantitative neuroimaging techniques has confirmed that the white matter of the brain is the most affected tissue by MS following a global pattern with greater involvement of the left hemisphere.
\end{abstract}

Keywords Pattern recognition, Multiple Sclerosis, Quantitative Magnetic Resonance Imaging.

\section{Introduction}

Multiple Sclerosis (MS) is a neurodegenerative disease characterized by inflammatory demyelination in the central nervous system, destroying the myelin sheath that surrounds the axons (Kritas et al., 2014). MS lesions are associated with inflammation and essential structural changes, such as demyelination and a variable extent of axonal destruction and scar formation. (Lassmann, 1999). Although the visible lesions in the medical images allow evaluating the evolution of the disease at a certain

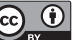

This is an Open Access article distributed under the terms of the Creative Commons Attribution License, which permits unrestricted use, distribution, and reproduction in any medium, provided the original work is properly cited.

How to cite this article: Pessini RA, Santos AC, Salmon CEG. Quantitative MRI data in Multiple Sclerosis patients: a pattern recognition study. Res Biomed Eng. 2018; 34(2):138-146. DOI: 10.1590/2446-4740.07117.

*Corresponding author: Center of Imaging Sciences and Medical Physics, Clinics Hospital, Ribeirão Preto Medical School, University of São Paulo, Av. Bandeirantes, 3900, Monte Alegre, CEP 14040-901, Ribeirão Preto, SP, Brazil. E-mail: rodpessini@gmail.com

Received: 08 November 2017 / Accepted: 01 May 2018 stage, the quantification of microscopic tissue damage can contribute to a better study of the disease and serve as basis for more specific and effective treatments (Filippi et al., 2017; Santos, 2007). MS is progressive and has no cure, there are only palliative treatments, which are partially effective, exist to manage the course of the disease and (Goldenberg, 2012).

Magnetic resonance imaging (MRI) is widely used for the diagnosis and treatment of neurodegenerative diseases by providing well-detailed images of brain structures. These images present good sensitivity to evidence demyelinating lesions, but low specificity in microscopic lesions. The great potential of intrinsic information in the MR signal from different pulse sequences led to the development of different quantitative methodologies contemplating biological aspects involved in the pathophysiological process of the production of the tissue lesion (Filippi et al., 2017; Santos, 2007). Since the early 1990s, more sophisticated MRI pulse sequences associated with post processing methods have been used to provide quantitative data that allow measuring non-visible microscopic changes in conventional images, therefore not accessible by conventional radiologist 
analysis. Among these quantitative techniques, the most known and widespread are: morphometry, relaxometry, magnetization transfer, proton spectroscopy and diffusion (Filippi et al., 2017).

Machine learning techniques are useful for exploring a hyperplane or hypersurface among high dimensional training features and the categorization of a testing subject as a member of a particular clinical group. For example, support vector machine has been used to differentiate Alzheimer's patients from normal controls and rule out alternative causes of dementia (Li et al., 2014). Pattern recognition techniques applied in neuroimaging data analysis may show greater sensitivity than traditional approaches such as statistical methods and visual inspection, contributing to the detection and treatment of diseases (Weygandt et al., 2011). Some recognition techniques can also be applied to identify the role of different characteristics in the classification problem. In other words, its application in a particular clinical group can indicate the more relevant parameters related to a particular disease.

Quantitative MRI techniques enable a comprehensive characterization of brain tissue, but generate a large number of numerical results. More than a thousand attributes can be generated from a single subject if a detailed segmentation of the brain is considered, making group studies complex and inefficient by parametric techniques of data analysis. A large volume of data can be manipulated using pattern recognition, a subtopic of machine learning which is in turn a subfield of artificial intelligence. In the neuroimaging area, the techniques of pattern recognition have gained space in the study of MS, but focused on the lesion's segmentation and on using attributes of shape and texture from qualitative anatomical images (Geremia et al., 2011; Kamber et al., 1995; Lao et al., 2008; Quddus et al., 2006). In 2011, Weygandt and colleagues extended the application of these techniques to areas of normal appearing white and gray matter using T2-weighted image data (Weygandt et al., 2011). In general, recognition techniques have reduced the number of false positives from visual evaluation in this particular application (Geremia et al., 2011; Lao et al., 2008; Weygandt et al., 2011). The use of data from quantitative MR techniques has been scarce because it requires the acquisition of a large number of subjects with several pulse sequences making the acquisition process complex and costly. Additionally, access to this data is generally restricted for privacy reasons. A recent work has shown the effectiveness of these recognition techniques in quantitative MRI data used in the study of Alzheimer's disease (Li et al., 2014). To the best of our knowledge, the use of pattern recognition in quantitative neuroimaging data from patients with MS has not been reported in the literature.
This approach should provide new information on how the classification of data can help in the diagnosis and treatment of MS, from quantitative techniques that allow measuring microscopic changes not visible in conventional images.

The general purpose of this study is to apply pattern recognition techniques to quantitative neuroimaging data acquired by magnetic resonance imaging in patients, especially in patients with Multiple Sclerosis. Previous studies have achieved an efficient separation of healthy control and patient groups from the neuroimaging data in other neurodegenerative diseases. Based on that, three are the main questions related to MS to be answered in this work: What are the main quantitative variables involved in the separation between groups? What are the major regions affected by the disease according to the classification tools? Are these major regions consistent with those recognized in the previous literature?

\section{Methods}

This study was approved by the ethics committee of the Clinics Hospital - Ribeirão Preto Medical School (process \#14124-15). Data from 144 clinically confirmed MS patients and 203 subjects with no detected neurological abnormalities, control subjects (CS), were used retrospectively. Images were acquired on a 3T magnetic resonance scanner (Achieva, Philips) from 2008 to 2014. Age interval was from 7 to 65 years for both groups (Table 1).

A survey was performed on the MRI reports of the MS patients to find out the severity of the disease in each case. The results were divided according to the degree of destructibility of the MS lesions: low, moderate and high. Among the MS patients considered in the sample $12 \%$ had lesions of low destructibility, $32 \%$ moderate and $56 \%$ high. Therefore, our patients sample was mainly constituted by individuals with the disease in moderate or advanced stage. Images from four MRI acquisitions were used: 3D-T1w, Diffusion Tensor Imaging (DTI), Magnetization Transfer (MT) and Transverse Relaxometry (T2). Detailed information about the selected acquisitions can be found in previous publications of our group (Carmo, 2014; Diniz et al., 2011). These acquisitions were chosen for the extraction of quantitative data with morphological and physiological interests, from the molecular-cellular to the anatomical-structural levels

Table 1. Demographic data (Age \& Sex) of our casuistic.

\begin{tabular}{lcccc}
\hline \multirow{2}{*}{ Group } & N & \multicolumn{2}{c}{ Age (years) } & Sex \\
\cline { 3 - 4 } & & Interval & $\begin{array}{c}\text { Average } \\
\text { (SD) }\end{array}$ & \\
(M/F) & \\
\hline MS Patients & 144 & $7-65$ & $41.5(11.6)$ & $44 / 100$ \\
Control Subjects & 203 & $7-65$ & $29.3(16.2)$ & $108 / 95$ \\
\hline
\end{tabular}


(Carmo, 2014). We defined 126 labels selected from 166 labels automatically segmented in the program FreeSurfer (version 4.5) (Stevens, 2017) using the recon-all function and Atlas Destrieux (Destrieux, 2010) in the 3D-T1w data. Our selection was based on the quality of the segmentation, avoiding labels attributed to very small volumes. Labels with volumes inferior to $0.2 \%$ of the intracranial volume were removed to avoid mis-segmented regions. Looking for a data consistency, the outliers were also removed considering z-scores less than 3. Some bilateral or subdivided brain regions were joined to one label to reach relative volumes superior to $0.2 \%$ (Table 2).

From the 3D-T1w data, cortical thickness and volume of each label were also extracted in FreeSurfer. Total intracranial volume of each individual was used to normalize the volumes in order to reduce the head size effect. From DTI data, fractional anisotropy (FA), mean diffusivity (MD), parallel diffusivity $(\mathrm{PaD})$ and perpendicular diffusivity $(\mathrm{PeD})$ maps generated on the scanner were used measurements. From MT and T2 acquisitions, Magnetization Transfer Ratio (MTR) and Transverse Relaxation Time (T2) maps were also generated, respectively, using locally developed functions based on MINC tools (Diniz et al., 2011). Quantitative information was extracted from each map and previously segmented 3D-T1w images (Figure 1). FA, MD, PeD and $\mathrm{PaD}$ data were only analyzed in white matter structures (58 labels). Cortical thickness was only evaluated in cortical regions with predominance of gray matter (52 labels). Other measurements were extracted in all the regions (Destrieux, 2010) (Table 2). All these measures defined the attributes of the characteristic vector for each subject.
The WEKA: Data Mining Software in Java (version 3.6.11) was used in mining and supervised classification tasks. The KNN classifier implemented in this program was chosen for its simplicity and robustness. Other classifiers might be could be more efficient, but a computational optimization of the classification procedure was not our main goal. Figure 1 shows the general flowchart of the procedure performed for the classification tasks.

In order to assess the relevance of each attribute (region plus technique); a previous selection of best attributes (BA) was performed with the attribute evaluator WrapperSubsetEval implemented in WEKA software. Different numbers of neighbors were considered in the $\mathrm{KNN}$ algorithm (1, 3 and 5 neighbors) looking to make independent the indication of the best attributes and the classification algorithm properties. An attribute ranking was performed considering the number of attribute indications among the five best attributes in each KNN algorithm using the global characteristic vector. Finally, the best attributes were selected looking for this ranking. To estimate the labels (cerebral regions) more involved in the classification procedure, a similar approach was followed but including the individual data of each technique. In this case, the ranking was performed counting the number of indications of each label disregarding the technique (for example: wm corpus callosum was indicated several times in different techniques in the folllowing attributes: FA wm corpus callosum, MTR_wm_corpus callosum, MD_wm_corpus callosum, PaD_wm_corpus callosum). In order to quantify the relevance of each brain region, a percentage value was calculated considering all the possible indications as best attribute, because some labels are not contained in all the techniques.

Table 2. Attributes joined to reach average volumes greater than $0.2 \%$.

\begin{tabular}{|c|c|c|}
\hline \multicolumn{2}{|c|}{ Previous Attributes } & \multirow{2}{*}{$\frac{\text { Later Attribute }}{\text { caudalanteriorcingulate }}$} \\
\hline lh-caudalanteriorcingulate & rh-caudalanteriorcingulate & \\
\hline lh-entorhinal & rh-entorhinal & Entorhinal \\
\hline lh-isthmuscingulate & rh-isthmuscingulate & isthmuscingulate \\
\hline lh-parahippocampal & rh-parahippocampal & parahippocampal \\
\hline lh-parsorbitalis & rh-parsorbitalis & Parsorbitalis \\
\hline lh-pericalcarine & rh-pericalcarine & Pericalcarine \\
\hline lh-rostralanteriorcingulate & rh-rostralanteriorcingulate & rostralanteriorcingulate \\
\hline lh-temporalpole & rh-temporalpole & Temporalpole \\
\hline$w m$ lh_caudalanteriorcingulate & wm_rh_caudalanteriorcingulate & wm_caudalanteriorcingulate \\
\hline wm_lh_corpuscallosum & wm_rh_corpuscallosum & wm_corpuscallosum \\
\hline$w m \_l h \_c u n e u s$ & $w m \_r h \_c u n e u s$ & wm_cuneus \\
\hline$w m \_l h \_$parahippocampal & wm_rh_parahippocampal & $w m \_$parahippocampal \\
\hline$w m$ lh_rostralanteriorcingulate & $w m$ rh_rostralanteriorcingulate & $w m \_$rostralanteriorcingulate \\
\hline Left-Pallidum & Right-Pallidum & Pallidum \\
\hline Left-Amygdala & Right-Amygdala & Amygdala \\
\hline CC_Posterior, Mid_Posterio & r, Central and Mid_Anterior & wm_corpuscallosum \\
\hline
\end{tabular}




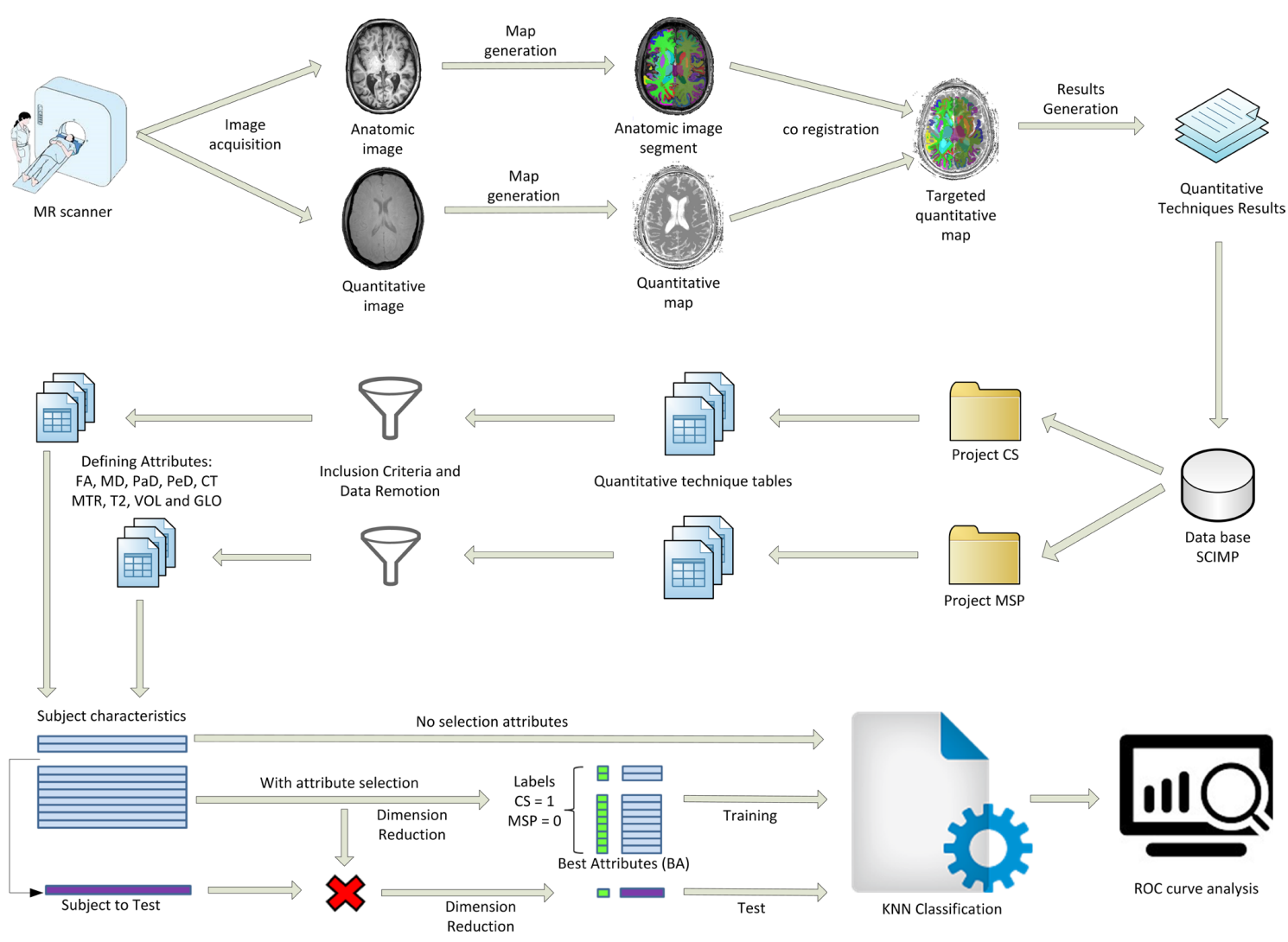

Figure 1. Flowchart of the procedure performed for the classification tasks from the MRI acquisitions to the efficiency assessment.

In order to identify the most relevant techniques for the classification procedure, two attributes selection strategies were followed: 1-Considering as attributes the individual data of each technique, 2-Using a global characteristics vector containing data of all the techniques. In this evaluation, the area under the ROC (Receiver Operating Characteristic) curve was the main parameter in the analysis. This area is estimated from the values of sensitivity and specificity in the confusion matrix. The number of instances changed for each classification because different subjects underwent different MRI acquisitions and some acquisitions were removed by quality assurance reasons.

Additionally, age and class (patient / control, to be only used in the training procedure) attributes were added in each vector of characteristics (Table 3). Age attribute was included to check its influence in the classification process due to the slight unbalance of the age between groups. Class attribute is necessary in the training procedure. To show the efficiency of the pre-selection of attributes, the same classifications were performed without considering this pre-selection (here called AA, all attributes). Figure 2 summarizes the classification tasks that were performed.
Table 3. Number of instances in the classifications considering the data of the individual and joint techniques.

\begin{tabular}{lccc}
\hline \multicolumn{1}{c}{ Data } & $\begin{array}{c}\text { Control } \\
\text { Subjects }\end{array}$ & $\begin{array}{c}\text { MS } \\
\text { Patients }\end{array}$ & Matrix \\
\hline FA (Dimensionless) & 75 & 90 & $165 \times 60$ \\
$\mathrm{MD}\left(\mathrm{mm}^{2} / \mathrm{s}\right)$ & 75 & 90 & $165 \times 60$ \\
$\mathrm{PaD}\left(\mathrm{mm}^{2} / \mathrm{s}\right)$ & 76 & 90 & $166 \times 60$ \\
$\mathrm{PeD}\left(\mathrm{mm}^{2} / \mathrm{s}\right)$ & 76 & 90 & $166 \times 60$ \\
$\mathrm{CT}(\mathrm{mm})$ & 167 & 144 & $311 \times 54$ \\
$\mathrm{MTR}(\%)$ & 91 & 109 & $200 \times 28$ \\
$\mathrm{~T} 2(\mathrm{~ms})$ & 90 & 108 & $198 \times 128$ \\
VOL $(\%)$ & 165 & 138 & $303 \times 128$ \\
GLO & 53 & 64 & $117 \times 664$ \\
\hline
\end{tabular}

Fractional Anisotropy (FA), Mean Diffusivity (MD), Parallel Diffusivity (PaD), Perpendicular Diffusivity (PeD), Cortical Thickness (CT), Magnetization Transfer Ratio (MTR), Transverse Relaxation Time (T2), Volumetry (VOL) and Global (GLO). The physical units are indicated in parentheses.

A final classification was made restricting to 53 the number of brain regions involved (Destrieux, 2010) (Table 2). This classification was considering the previous findings of the literature based on parametric statistics (Santos, 2007) and the clinical indication of a neuroradiologist with more than 25 years of clinical experience (A.C.S.). This restricted classification was 
also performed following the flowchart presented in Figure 1 with the same subjects, but using only five neighbors in the KNN algorithm (KNN5). Classifications with all labels and reduced number of labels, here called main (MC) and restricted (RC), respectively, were finally compared taking into account the area under the ROC curve (AUC). In this comparison, only the KNN5 algorithm with and without pre-selection of better attributes was considered.

\section{Results}

Two attributes were the most indicated in the previous selection of better attributes in the separation of CS and MS patients considering all data: VOL wm hypointensities and MTR wm corpuscallosum. Figure 3 shows the mean and standard deviation values of these attributes in the data from healthy subjects and MS patients.

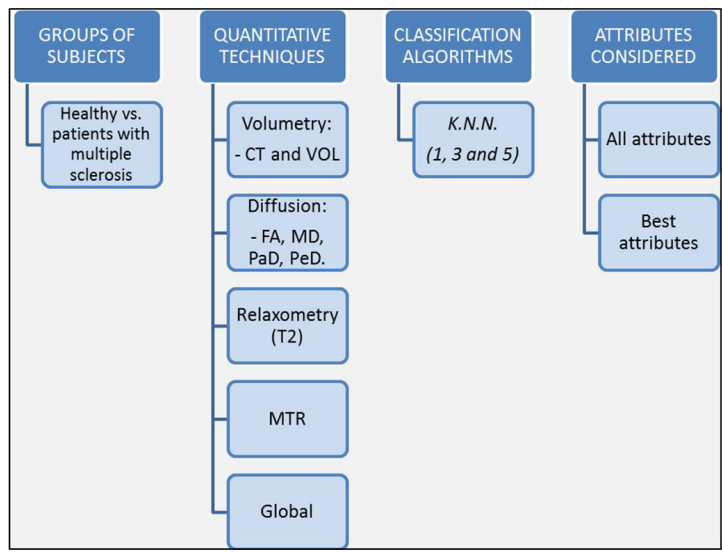

Figure 2. Representative scheme of classification tasks performed indicating the data and algorithms considered in each classification.

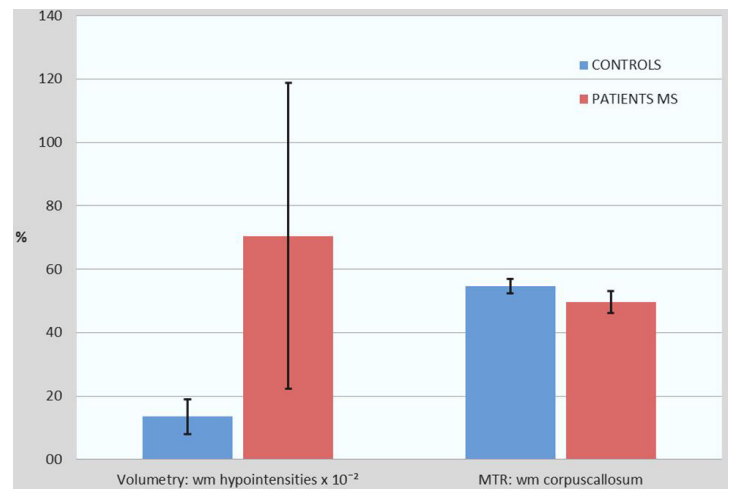

Figure 3. Mean values of the best attributes obtained in the attribute pre-selection considering the global vector in the control and patient groups. The standard deviation values are also indicated in the graph. The vertical axis should be viewed with caution, since each attribute indicates values with different meanings.
Both attributes showed significant differences between groups in an unpaired t test $(\mathrm{p}<0.001)$.

Figure 4 shows the labels with the highest percentage of indications among the best attributes grouped by labels for main and restricted classifications.

As shown in Figure 4, the main and restricted rankings had several common regions among the five best attributes of each classification. The wm corpuscallosum appeared in the top position in both classifications (MC: $30 \%$ and RC: $36 \%$ ), being considered the region most affected by MS, containing the best attributes for class separation. The wm hypointensities (MC: 17\% and RC: 24\%) and left cerebellum white matter (MC: $16 \%$ and RC: 12\%) were highlighted by appearing in both classifications. The regions $\mathrm{wm} \mathrm{lh}$ precuneus and wm lh fusiform were not used in RC, but appeared in 2 nd and 4 th positions with $20 \%$ and $17 \%$, respectively, in the $\mathrm{MC}$ ranking.

Figure 5 shows the AUROC values of the main classification using the KNN5 algorithm, with previous selection of attributes and considering the techniques separately and together. The choice of the algorithm with five neighbors was due to the better results when compared to those obtained with a smaller number of neighbors. As expected, the overall vector presented the highest percentages of AUROC reaching 97.1\%. In addition, according to the graph, the quantitative

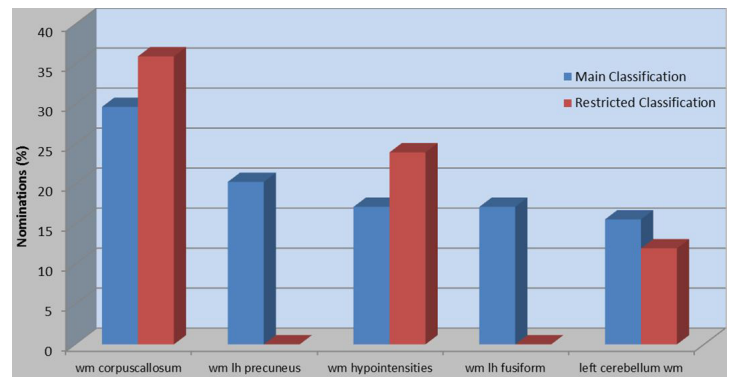

Figure 4. Labels with the highest number of nominations among the five best attributes in the main and restricted classifications.

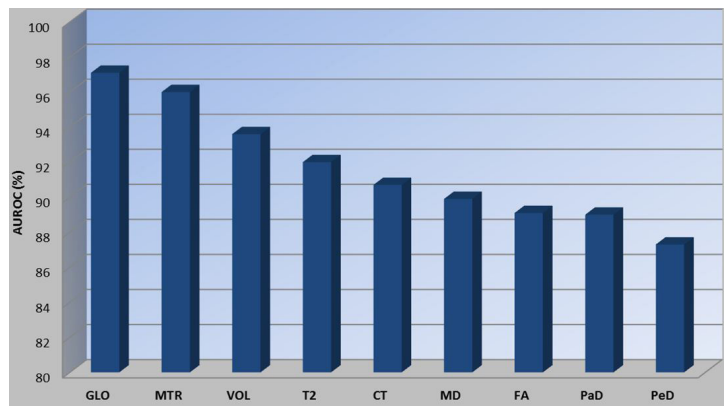

Figure 5. Areas under the ROC curve in the classifications with attribute pre-selection considering different input data in the main classification and using the KNN5 algorithm. 
technique with inferior performance in this classification was the perpendicular diffusibility with values below $90 \%$ and the best quantitative technique was the magnetization transfer reaching $96 \%$.

Table 4 shows the accuracy values obtained in the different classifications using the KNN5 algorithm. As expected, the results followed the same trend as the AUROC values in the main classification (Figure 6) considering the quantitative data involved. Additionally, a quantitative AUROC improvement (2-10\%) can be observed with the use of the best attributes (BA). In general, the mean accuracy values of the main classification were slightly higher than those for the restricted classification; however, the difference was small, showing equivalence between both classifications (MC and RC).

Table 4. Accuracy values (\%) for the main (all regions) and restricted (selected regions) classifications using KNN5 and considering different input data with (Best attributes) and without (All attributes) pre-selection of attributes.

\begin{tabular}{ccccccc}
\hline \multirow{2}{*}{ Classification } & & \multicolumn{2}{c}{ All regions } & & \multicolumn{2}{c}{ Selected regions } \\
\cline { 1 - 3 } \cline { 6 - 7 } Attributes & & All & Best & & All & Best \\
\cline { 1 - 3 } GLO & & 84.6 & 95.7 & & 83.8 & 93.2 \\
MTR & & 81.0 & 88.0 & & 83.0 & 87.0 \\
VOL & & 82.2 & 87.5 & & 84.8 & 91.7 \\
CT & & 81.7 & 85.2 & & 76.5 & 77.8 \\
T2 & 75.8 & 86.9 & & 78.3 & 81.8 \\
FA & 82.4 & 84.8 & & 70.3 & 84.8 \\
MD & & 80.6 & 84.2 & & 80.6 & 86.1 \\
PaD & & 77.1 & 81.3 & & 69.3 & 81.3 \\
PeD & 79.5 & 81.3 & & 76.5 & 85.5 \\
\hline
\end{tabular}

Global (GLO), Magnetization Transfer Ratio (MTR), Volumetry (VOL), Cortical Thickness (CT), Transverse Relaxation Time (T2), Fractional Anisotropy (FA), Mean Diffusivity (MD), Parallel Diffusivity (PaD) and Perpendicular Diffusivity (PeD).

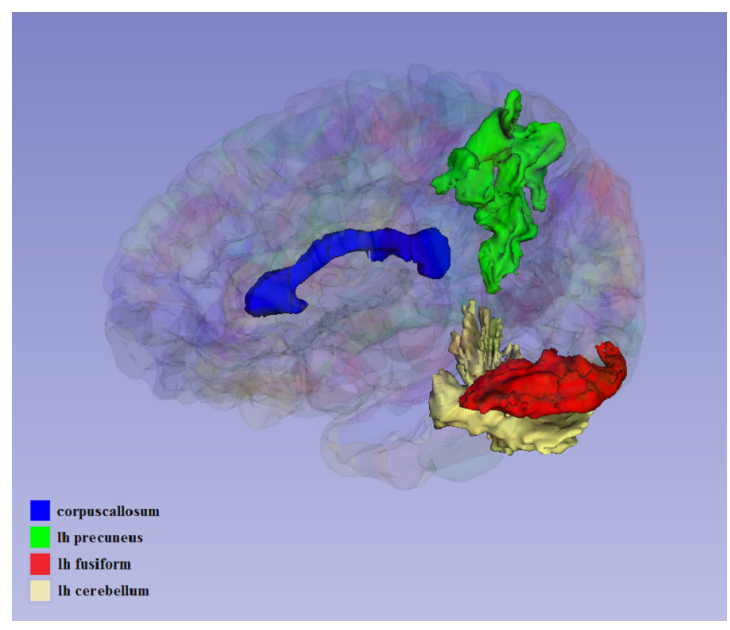

Figure 6. 3D model of the brain indicating the location of the four most indicated regions among the best attributes in the main classification.

\section{Discussion}

From the data provided by all techniques, the best attributes for the identification of MS patients were: VOL wm hypointensities and MTR wm corpuscallosum (Figure 3). In the VOL wm_hypointensities attribute the mean difference in normalized volume between MS and $\mathrm{CN}$ patients reached $0.60 \%$, being a strong indicator of the difference between groups (Figure 3). The high standard deviation of the wm_hypointensities label in patients $(0.51 \%)$ suggests individuals at different stages of the disease. This specific label does not represent an anatomical region in the FreeSurfer segmentation but non-specific areas in the white matter, possibly representative of demyelinating lesions. Hence, this label could be used as an initial guess in a MS lesion segmentation tool.

The corpus callosum is the cerebral structure with the highest concentration of inter-hemispheric axonal fibers. Integrity of the axonal fibers can be evaluated from MTR values. Reduced values of MTR in patients are a consequence of the demyelination process (Harrison et al., 2013). In our case, MTR in corpus callosum present a reduced value in patients, as expected. It should be noted that the use of parametric statistics would reveal differences between patients and healthy controls in several attributes, which hinders the interpretation of the results, even more because of their multifactorial nature. However, the use of machine learning techniques helps to identify the key cerebral regions and quantitative techniques related to the disease.

In order to find the main regions affected by the disease according to the classification tools, the attributes pre-selection results were grouped by regions independently of the quantitative technique involved (Figure 4). The corpus callosum region and wm_hypointensities label were the most frequent areas (above 20\%) in the selection of the best attributes in the restricted classification. Another region indicated as a relevant region was the white matter in the left cerebellum; this region had previously been reported with significant alterations in MS patients (Reuter et al., 2009).

Two additional WM regions were highlighted in the main classification: left precuneus and left fusiform (Figure 4). These areas have not been frequently reported in MS patient's studies. In general, labels located in different and distant areas of the white matter were indicated as more relevant regions in the discrimination process between healthy individuals and patients with MS. Figure 6 shows the anatomical regions most involved in the selection of the best attributes in the separation between patients and controls, highlighting the non-focal nature of the disease. 
There are currently a large number of quantitative MRI techniques available. Thus, when defining a research protocol for the study of a certain disease, it is extremely important to define a priori the most relevant techniques for the clinical question in order to save resources and reduce patient's time in the scanner. Our results indicate that the use of the magnetization transfer technique presents advantages when compared to the results of the other techniques in the identification of MS patients (Figure 5). The classification problem using these data can be consider an easy problem for the algorithm, because even using only diffusion-weighted images AUROC value exceeds $86 \%$. This fact does not indicate that the quantitative changes can be visually detected for two main reasons: the radiologist often does not have access to all the quantitative maps and the number of regions to be evaluated is very high.

As expected, the combined use of all techniques resulted in an excellent 98\% AUROC using KNN5 combined with a pre-selection of attributes. Considering the accuracy instead AUROC as a comparison metric, the results between the techniques are almost identical (second column Table 4). In this case, the use of all quantitative neuroimaging results allowed an accuracy of $95.7 \%$. All these results confirm that the use of the machine learning tool in these data is a great alternative in the classification of MS patients for medical training purposes. This tool can be used to train medical residents in the identification of MS patients. The problem may be relatively easy for an experienced radiologist, but not for a student.

From Table 4, it can be noticed that there is a significant reduction of accuracy when a pre-selection of attributes is not made. On the other hand, the use of a restricted classification considering less brain areas brought a slight accuracy decrease, less than $2 \%$ in most cases, except for the cortical thickness.

The results are different for each technique, according to the specifications of each physical phenomenon and the errors involved in the estimation of each quantitative parameter. In general, the white matter regions have a reduction in the MTR (Figure 3) and FA values, and an increase in the diffusion coefficients (MD, $\mathrm{PaD}$ and $\mathrm{PeD}$ ) in the patient group. The above findings are indicative of the well-known myelinic damage in several WM regions (Filippi et al., 2017; Kritas et al., 2014; Papathanasiou et al., 2017; Reuter et al., 2009; Santos, 2007; Vollmer et al., 2015). By other hand, our common finding in gray matter regions of the patients was the increase of the atrophy of cortical and subcortical structures, also in agreement with previous reports (Narayana et al., 2012; Steenwijk et al., 2016). T2 increase was observed in structures containing white and gray matter, but it was predominant and diffuse in the white matter, similar to a previous report (Neema et al., 2009). Reduction of MTR values in patients means demyelination and axonal injury in WM and plasma membrane loss in GM (Kucharczyk et al., 1994). The presence of demyelinating lesions modifies the proportions of free and bound water influencing the magnetization transfer mechanism. WM regions showed a widespread MTR reduction; however, some GM regions also showed changes in MS patients, as reported by Cercignani and collaborators (Cercignani et al., 2001). In our study, classification techniques identified left hippocampus as a predominantly GM region with relevant variations in MTR values.

Water diffusion is affected by the properties of the medium where the molecular movement occurs; hence, the diffusion measurements in the biological tissues provide information on the structures of the tissues. Water movement can be hampered by the presence of structural barriers at cellular levels. Pathological processes can alter the organization of tissue, causing abnormalities in water diffusion (Filippi et al., 2001). The classification results considering diffusion data are in agreement with the expected results. FA values were higher in CS than in the MS group, indicating loss of the axonal integrity in WM regions. The values of diffusibility showed an inverse behavior, i.e., highest values for MS patients. Pathological elements of MS have the potential to alter the permeability or geometry of structural barriers to the molecular diffusion of water in the brain (Filippi et al., 2001). The accumulation of inflammatory cells and the decomposition of myelin products could potentially restrict water diffusion and decrease fractional anisotropy because of the presence of diffusion barriers. WM regions are the areas most affected by MS, possibly causing diffuse hyperplasia, uneven edema, perivascular infiltration, gliosis, abnormally thin myelin and axonal loss (Filippi et al., 2001). Diffusion changes in WM are expected in a widespread way, but tend to be more severe in places such as periventricular areas and WM of the frontal lobe (Filippi et al., 2001). In our study, diffusion changes were also found in the frontal lobe of the WM, as expressed by the mean values of FA (lh medial and frontal hemispheres: MS $0.28 \mathrm{~mm}^{2} / \mathrm{s}$, CS $0.31 \mathrm{~mm}^{2} / \mathrm{s}$ ), $\mathrm{PaD}$ (lh medial and frontal hemispheres: MS $0,92 \mathrm{~mm}^{2} / \mathrm{s}$, CS $0.83 \mathrm{~mm}^{2} / \mathrm{s}$ ) and $\mathrm{PeD}$ (lh lateral orbito frontal: MS $0.70 \mathrm{~mm}^{2} / \mathrm{s}$, CS $0.65 \mathrm{~mm}^{2} / \mathrm{s}$ ).

Our results indicate a reduced mean cortical thickness in all considered cortical structures in MS patients when compared to healthy individuals, suggestive of a volume atrophy of the cortex due to MS progression, in agreement to a previous study (Sailer et al., 2003). Focal thinning was also observed in frontal (left precentral: MS $2.45 \mathrm{~mm}$, CS $2.55 \mathrm{~mm}$ ) and temporal regions (left fusiform: MS $2.65 \mathrm{~mm}$, CS $2.95 \mathrm{~mm}$ ). Increased 
lesion volume causes irreversible tissue loss leading to neuronal loss with axonal transection and subsequent axonal injury of cortical neurons in multiple sclerosis (Sailer et al., 2003).

Considering the volume variations, we did not find a significant volume loss rate compared to normal aging, as concluded by a recent meta-analysis (Vollmer et al., 2015). Our volume results indicate focal losses in some structures, e.g. left lateraloccipital: MS 0.73\%, CS $0.94 \%$ and right ventral: MS $0.27 \%$, CS $0.31 \%$. Some authors suggest that the most pronounced decrease in the volume of the brain of MS patients occurs in the early phase of the disease, where there is a higher inflammatory level of the disease. In our sample, only $12 \%$ of the patients were in an initial phase, it may be for this reason that volumetry was not indicated as the best individual technique, although its accuracy is more than $87 \%$ (Table 4).

Our T2 results indicate that lesions caused by MS increased the concentration of water in the tissues suggesting the presence of edema, demyelination, gliosis or axional dysfunctions. A previous study (Neema et al., 2009) indicated T2 prolongation in MS compared to CS in the whole brain, frontal normal-appearing white matter (NAWM), parietal NAWM and callosal genu. Our study corroborated these findings, because the corpus callosum and left fusiform were chosen among the regions having the best attributes. In general, our findings suggest a substantial loss of the axonal fibers of the corpus callosum in a patient with MS.

In summary, the use of MTR, structural morphometry, relaxometry and diffusion data combined with a simple classification algorithm (KNN) allowed the definition of a classification strategy with more than $95 \%$ accuracy in the separation of MS patients and healthy subjects. According to our results, the quantitative technique with the highest discrimination power was the MT and diffusion weighted imaging obtained the lowest results, particularly the perpendicular diffusibility parameter. Consistent with the literature, our findings confirm reduction of MTR, volume, cortical thickness, FA, and diffusivity; but an increase in the T2 values in several areas of the patients' brains (Filippi et al., 2017). In general, the techniques showed that MS causes: demyelination, WM lesions, edema, axonal loss, gliosis and loss of brain volume.

Our results reinforce the idea that different regions of the brain are affected by MS, especially in WM. Although, the changes are diffuse, with some specific regions more involved in the subject's classification: corpus callosum, left cerebellum, left precuneus and left fusiform. These two latter areas had not been described as being particularly affected by the disease, but the use of pattern recognition techniques helped to identify them. Classification techniques proved to be a suitable tool for discrimination between healthy individuals and MS patients, also indicating the best attributes resulting from quantitative evaluations, being consistent with expected results from the pathophysiology of the disease.

\section{Acknowledgements}

The authors are grateful to funding agencies $\mathrm{CNPq}$ (grant number 311703/2014-3) and FAPESP (process 05/56447-7). We also thank Dr. Antonio Carlos da Silva Senra Filho for his useful suggestions and comments.

\section{References}

Carmo SS. Características do envolvimento do Sistema Nervoso Central na Polirradiculoneuropatia Inflamatória Desmielinizante Crônica: um estudo mediante técnicas quantitativas de Imagem por Ressonância Magnética [thesis]. Ribeirão Preto: Universidade de São Paulo; 2014.

Cercignani M, Bozzali M, Iannucci G, Comi G, Filippi M. Magnetisation transfer ratio and mean diffusivity of normal appearing white and grey matter from patients with multiple sclerosis. J Neurol Neurosurg Psychiatry. 2001; 70(3):311-7. http://dx.doi.org/10.1136/jnnp.70.3.311. PMid:11181851.

Destrieux C. Destrieux atlas changes [Internet]. Cambridge: Harvard University; 2010. [cited 2010 Feb 28]. Available from: https://surfer.nmr.mgh.harvard.edu/fswiki/DestrieuxAtlasChanges

Diniz PRB, Velasco TR, Salmon CEG, Sakamoto AC, Leite JP, Santos AC. Extratemporal damage in temporal lobe epilepsy: magnetization transfer adds information to volumetric MR imaging. AJNR Am J Neuroradiol. 2011; 32(10):1857-61. http://dx.doi.org/10.3174/ajnr.A2639. PMid:21885719.

Filippi M, Cercignani M, Inglese M, Horsfield MA, Comi G. Diffusion tensor magnetic resonance imaging in multiple sclerosis. Neurology. 2001; 56(3):304-11. http://dx.doi. org/10.1212/WNL.56.3.304. PMid:11171893.

Filippi M, Preziosa P, Rocca MA, Microstructural MR. Microstructural MR imaging techniques in multiple sclerosis. Neuroimaging Clin N Am. 2017; 27(2):313-33. http://dx.doi. org/10.1016/j.nic.2016.12.004. PMid:28391789.

Geremia E, Clatz O, Menze BH, Konukoglu E, Criminisi A, Ayache N. Spatial decision forests for MS lesion segmentation in multi-channel magnetic resonance images. Neuroimage. 2011; 57(2):378-90. http://dx.doi.org/10.1016/j.neuroimage.2011.03.080. PMid:21497655.

Goldenberg MM. Multiple sclerosis review. Pharm Ther. 2012; 37(3):175-84. PMid:22605909.

Harrison DM, Shiee N, Bazin PL, Newsome SD, Ratchford JN, Pham D, Calabresi PA, Reich DS. Tract-specific quantitative MRI better correlates with disability than conventional MRI in multiple sclerosis. J Neurol. 2013; 260(2):397-406. http:// dx.doi.org/10.1007/s00415-012-6638-8. PMid:22886062.

Kamber M, Shinghal R, Collins DL, Francis GS, Evans AC. Model-based 3-D segmentation of multiple sclerosis lesions in magnetic resonance brain images. IEEE Trans Med Imaging. 
1995; 14(3):442-53. http://dx.doi.org/10.1109/42.414608. PMid: 18215848 .

Kritas SK, Saggini A, Cerulli G, Caraffa A, Antinolfi P, Pantalone A, Rosati M, Tei M, Speziali A, Saggini R, Frydas A, Conti P. Impact of mast cells on multiple sclerosis: Inhibitory effect of natalizumab. Int J Immunopathol Pharmacol. 2014; 27(3):331-5. http://dx.doi.org/10.1177/039463201402700303. PMid:25280024.

Kucharczyk W, Macdonald PM, Stanisz GJ, Henkelman RM. Relaxivity and magnetization transfer of white matter lipids at MR imaging: Importance of cerebrosides and $\mathrm{pH}$. Radiology. 1994; 192(2):521-9. http://dx.doi.org/10.1148/ radiology.192.2.8029426. PMid:8029426.

Lao Z, Shen D, Liu D, Jawad AF, Melhem ER, Launer LJ, Bryan RN, Davatzikos C. Computer-assisted segmentation of white matter lesions in 3D MR images using support vector machine. Acad Radiol. 2008; 15(3):300-13. http://dx.doi. org/10.1016/j.acra.2007.10.012. PMid:18280928.

Lassmann $H$. The pathology of multiple sclerosis and its evolution. Philos Trans R Soc Lond B Biol Sci. 1999; 354(1390):1635-40. http://dx.doi.org/10.1098/rstb.1999.0508. PMid:10603616.

Li M, Qin Y, Gao F, Zhu W, He X. Discriminative analysis of multivariate features from structural MRI and diffusion tensor images. Magn Reson Imaging. 2014; 32(8):1043-51. http://dx.doi.org/10.1016/j.mri.2014.05.008. PMid:24970026.

Narayana PA, Govindarajan KA, Goel P, Datta S, Lincoln JA, Cofield SS, Cutter GR, Lublin FD, Wolinsky JS. Regional cortical thickness in relapsing remitting multiple sclerosis: a multi-center study. Neuroimage Clin. 2012; 2:120-31. PMid:24179765.

Neema M, Goldberg-Zimring D, Guss ZD, Healy BC, Guttmann CR, Houtchens MK, Weiner HL, Horsfield MA, Hackney DB, Alsop DC, Bakshi R. 3T MRI relaxometry detects T2 prolongation in the cerebral normal-appearing white matter in multiple sclerosis. Neuroimage. 2009; 46(3):633-41. http:// dx.doi.org/10.1016/j.neuroimage.2009.03.001. PMid:19281850.

Papathanasiou A, Messinis L, Zampakis P, Papathanasopoulos P. Corpus callosum atrophy as a marker of clinically meaningful cognitive decline in secondary progressive multiple sclerosis. Impact on employment status. J Clin Neurosci. 2017; 43:170-5. http://dx.doi.org/10.1016/j.jocn.2017.05.032. PMid:28601572.
Quddus A, Fieguth P, Basir O. Adaboost and Support Vector Machines for white matter lesion segmentation in MR images. In: IEEE-EMBS 2005: Proceedings of the 27th Annual International Conference of the Engineering in Medicine and Biology Society; 2006 Jan 17-18; Shanghai, China. USA: IEEE; 2006. vol. 1, p. 463-6. http://dx.doi.org/10.1109/ IEMBS.2005.1616447.

Reuter F, Del Cul A, Malikova I, Naccache L, Confort-Gouny S, Cohen L, Cherif AA, Cozzone PJ, Pelletier J, Ranjeva J-P, Dehaene S, Audoin B. White matter damage impairs access to consciousness in multiple sclerosis. Neuroimage. 2009; 44(2):590-9. http://dx.doi.org/10.1016/j.neuroimage.2008.08.024.

Sailer M, Fischl B, Salat D, Tempelmann C, Schönfeld MA, Busa E, Bodammer N, Heinze HJ, Dale A. Focal thinning of the cerebral cortex in multiple sclerosis. Brain. 2003; 126(Pt 8):1734-44. http://dx.doi.org/10.1093/brain/awg175. PMid:12805100.

Santos AC. Quantificação das mudanças no encéfalo causadas pela Esclerose Múltipla usando Ressonância Magnética Quantitativa [thesis]. Ribeirão Preto: Universidade de São Paulo; 2007.

Steenwijk MD, Geurts JJ, Daams M, Tijms BM, Wink AM, Balk LJ, Tewarie PK, Uitdehaag BM, Barkhof F, Vrenken H, Pouwels PJ. Cortical atrophy patterns in multiple sclerosis are non-random and clinically relevant. Brain. 2016; 139(Pt 1):11526. http://dx.doi.org/10.1093/brain/awv337. PMid:26637488.

Stevens A. FreeSurferWiki [Internet]. Cambridge: Harvard University; 2017. [cited 2017 Out 24]. Available from: https:// surfer.nmr.mgh.harvard.edu/fswiki

Vollmer T, Signorovitch J, Huynh L, Galebach P, Kelley C, DiBernardo A, Sasane R. The natural history of brain volume loss among patients with multiple sclerosis: a systematic literature review and meta-analysis. J Neurol Sci. 2015; 357(1-2):8-18. http://dx.doi.org/10.1016/j.jns.2015.07.014. PMid:26238166.

Weygandt M, Hackmack K, Pfuller C, Bellmann-Strobl J, Paul F, Zipp F, Haynes JD. JD. MRI pattern recognition in multiple sclerosis normal-appearing brain areas. PLoS One. 2011; 6(6):e21138. http://dx.doi.org/10.1371/journal.pone.0021138. PMid:21695053. 